\title{
Cation and anion contributions to gustatory quality of simple salts
}

\author{
ERNEST DZENDOLET AND HERBERT L, MEISELMAN
}

UNIVERSITY OF MASSACHUSETTS

Curves of mean percentages of the four gustatory quality responses as a function of concentration of solutions of $\mathrm{LiCl}$, $\mathrm{KCl}$, lithium sulfate, and potassium sulfate were evaluated as to the relative contributions of the cation and anion to the quality. It was concluded that chloride ion, and sulfate ion to a lesser degree, were responsible for the salty quality. In the mid-range concentrations, potassium ion was responsible for the bitter quality, and lithium ion, by means of an ion-solvent interaction; was responsible for the sour quality reported. At the very low concentrations, at which all salts tested evoked sweet responses, a structure produced by a solvent-cation interaction was assumed to be responsible. The details of the presumed structure are still to be clarified. Although one ion in each salt is primarily responsible for a particular quality over a given concentration, the other ion usually modifies the effect.

Höber and Kiesow (1898) were apparently the first to assume that the individual cations and anions may contribute separate gustatory qualities to the overall taste of a salt. They indicated that the difficulties inherent in such identification were that (1) a salt solution can change its quality as a function of its concentration, and (2) a salt solution at a given concentration can have a number of qualities. To avoid these difficulties, Höber and Kiesow restricted their Ss to the. use of the salty response alone, and determined the absolute threshold (RL) for this quality for a number of salts. If the RL for salty occurred at about the same concentration of the same ion in different salts, then this lon was the one presumed to be responsible for the salty quality. The test solutions were kept at $25^{\circ} \mathrm{C}$, and were presented in order of increasing concentration, i.e., method of limits, ascending series only. Two cubic centimeters of solution were flowed over the tongue from a pipette, and the tongue was rinsed with water between presentations. Höber and Klesow served as their own Ss.

The authors concluded that the salty quality was caused by the halides tested, i.e., $\mathrm{Cl}^{-}, \mathrm{I}^{-}$, and $\mathrm{Br}^{-}$, and also by sulfate, although the salty quality of the sulfate was very difficult to detect because of other qualities present. The RL concentration at the tongue tip for the above anions ranged between 0.02 and $0.04 \mathrm{M}$. Some beryllium salts were also tested and reported as evoking sweet at the lower concentrations used, and sour-sweet at the higher ones. Höber and Kiesow assumed that the sweet quality was caused by this cation. The RL for this quality ranged between about 0.0002 and $0.0005 \mathrm{M}$ at the tongue tip. Bases were also investigated, and found to have an RL for sweet ranging from about 0.0005 to $0.0008 \mathrm{M}$. The hydroxyl ion was assumed to be the cause of sweet in these compounds. Sodium or potassium ions did not appear to evoke any distinct quality.

Renquist (1919) repeated and extended the work of Höber and Kiesow (1898), by investigating most of the alkali halides, e.g., lithium bromide, and three alkaline earth chlorides, e.g., magnesium chloride. He also used the method of limits, but a 4-cc volume of distilled water was sipped first and expelled, and then a 4-cc volume of the test solution was sipped. The quality of the test solution as compared with the distilled water was then reported. Renquist mostly confirmed the earlier finding of quality change with concentration, but also found that potassium salts had a bitter taste rather than none at medium concentrations. This quality was in addition to the sweet and salty qualities found earlier at lower and higher concentrations, respectively. The alkaline earth chlorides were reported as evoking about the same qualities except that, at the low concentrations, they evoked either a bitter or inslpid quality in addition to the sweet. Renqvist also confirmed the earlier finding that potassium hydroxide tasted sweet from its threshold to about $0.01 \mathrm{M}$, at which concentration a "sharp" or "biting" sensation occurred. In addition, Renqvist found that potassium fluoride evoked sweet at the low concentrations, sour at the medium ones, and salty-sour at the high ones.

Renqvist concluded that the hydroxyl ion was responsible for the sweet quality, at least in inorganic compounds, and that the sweet quality at low concentrations of inorganic salts was due to the presence of hydroxyl ions. These hydroxyl ions were supposedly produced by a hydrolysis reaction between the salt and water of the solution. Unfortunately, this process is now known not to occur with the salts used by Renqvist. Because of this conclusion concerning the sweet quality, Renqvist considered the sweet taste an artifact, and assumed that the typical quality of the salt occurred in its mid-range concentration, i.e., above the concentration at which sweet was evoked. He hypothesized that the quality of a salt in this range was related to the relative ionic mobilities of the cation and anion. For example, a salt such as $\mathrm{KCl}$, with almost equal mobilities of cation and anion, would evoke bitter. 
This assumption did not allow the attributing of specific qualities to different ionic species as Höber and Kiesow had done, but maintained that the quality of a salt was some function of the cation-anion relationship.

Kionka and Strätz (1922) were also interested in the contribution of lons to gustatory quality. They investigated 18 alkall halides, e.g., LiBr, RbI, etc., and determined (1) the absolute threshold, and (2) the qualities evoked by $0.01 \mathrm{M}$ solutions. The solutions were kept at "body temperature," and distilled water at the same temperature was used to rinse the mouth after each solution presentation. Four Ss were employed and the psychophysical method of limits, with descending series only, was used. The initial concentration was the $0.01 \mathrm{M}$ solution. The solution was trickled over the tongue from a graduated burette until $S$ reported whether or not the taste was present. The amount of solution used was variable, being less at the higher concentrations, about $0.5 \mathrm{cc}$, and more at the lower concentrations, from 6 to $10 \mathrm{cc}$. Kionka and Strätz concluded on the basis of the different qualities reported to the various alkali halides that, to a first approximation, the intensity is determined by the cation, and the quality by the anion. For example, all the chlorides evoked primarily salty responses with perhaps a slight bitter aspect for some of them.

The paper presented here is based on data presented earlier (Dzendolet \& Meiselman, 1967) for a different purpose. These data have now been replotted and subjected to an analysis concerned with the contribution of the individual ions to the overall quality of a salt. The analysis is based on the inspection of curves of percentage responses of a particular quality as a function of concentration of a given salt. The basic assumption is that if a particular ion is responsible for a particular quality, then the curves of the percentage of responses of that quality, for all salts containing that ion, will be similar to one another in general form and will occur within the same general concentration range.

\section{Method}

For a detailed description of the method, see Dzendolet and Meiselman (1967). In essence, four undergraduate female $\mathrm{Ss}$ were selected from a group of 23 on the basis of a certain criterion of correct responding to four screening solutions which represented the gustatory qualities of bitter, salty, sour, and sweet. The test solutions for the main experiment were seven concentrations of lithium and potassium chlorides ranging from 0.004 to $0.05 \mathrm{M}$, and lithium and potassium sulfates ranging from 0.002 to 0.025 F. A discussion of the use of molar (M) and formal (F) concentration units has also been given earlier (Dzendolet \& Meiselman, 1967).

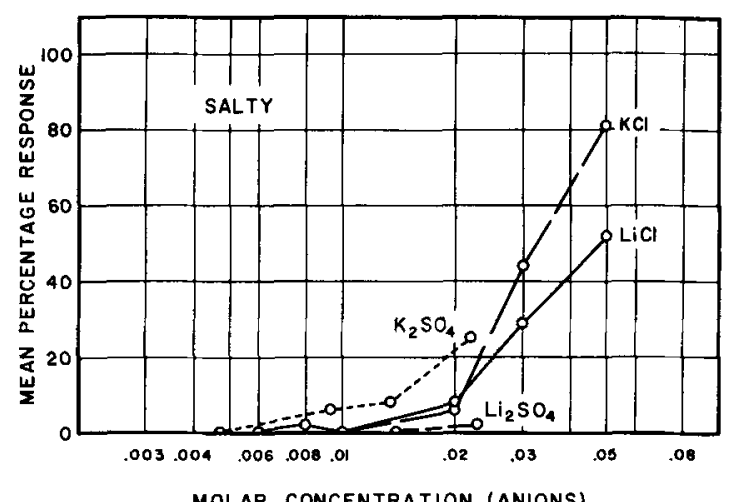

Fig. 1. Mean percentages of salty responses, from four selected female $\mathrm{Ss}$, as a function of anion concentration of the four indicated inorganic saits.

\section{Results}

Figure 1 shows the mean percentage of salty responses as a function of the anion concentration for each of the four salts tested. The responses were plotted in this way because of the earlier conclusions by Höber and Kiesow (1898) and Kionka and Strätz (1922) that the chlorides and sulfates were primarily responsible for the salty quality. Because the sulfates are not completely dissociated, as are the chlorides, the anion concentrations of these two salts had to be calculated. The dissociation constants in Harned and Owen (1950, Table 63-2) were used. It can be seen that the slopes of the increase in the salty percentages given to the chlorides and to potassium sulfate are about the same. The increase in responses to lithium sulfate, on the other hand, is very small. Because higher concentrations of these sulfates were not used, it is not certain whether or not responses to potassium sulfate would continue to parallel those of the chlorides, and whether or not responses to lithium sulfate would finally increase. A plot of these data as a function of cation concentration would merely move the curve for potassium sulfate over to the right, with the next-to-last point at about $0.028 \mathrm{M}$ and the last at about $0.047 \mathrm{M}$.

Figure 2 shows the mean percentages of bitter responses as a function of cation concentration. In all these figures, unless there was a theoretical reason for using the anion concentration as the abscissa, as with the data in Fig. 1, the cation concentration was used. The two lithium compounds show a very low percentage of responses, whereas the two potassium salts show a high level of responses. The $\mathrm{KCl}$ peaks sharply at $0.02 \mathrm{M}$, whereas the potassium sulfate shows more of a plateau.

In Fig. 3, the mean percentages of sour responses are shown against cation concentration. In this figure, it is the lithium compounds which are associated with a high percentage of responses, and the 


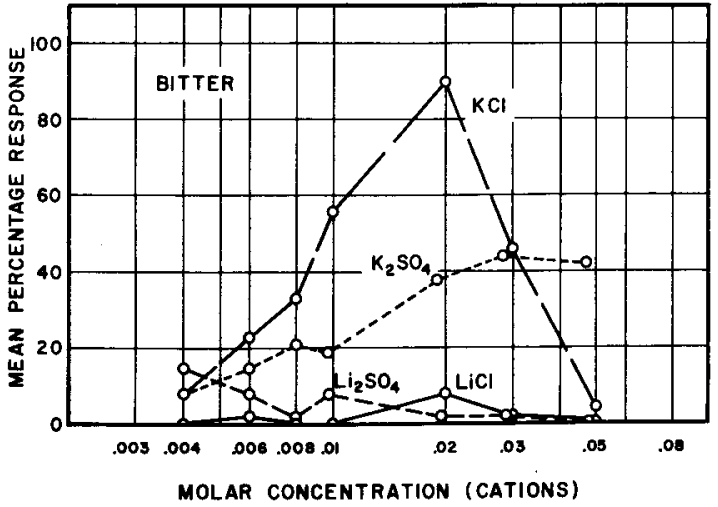

Fig. 2. Mean percentages of bitter responses plotted against cation concentration.

potassium compounds with a relatively low level of responses, although there appears to be an orderly rise at higher concentrations with the potassium sulfate. With this quality, it is the LiCl which shows a sharp peak, and the lithium sulfate which shows a slower rise to a plateau. The situation is similar to that indicated by the curves for the bitter quality, except for the difference in the cations in the two cases.

Figure 4 shows the sweet responses, combined with a few responses of no taste, as a function of cation concentration. It can be seen that there is no major difference between these curves except for a tendency for the chloride curves to be parallel to one another, as are the sulfate curves to one another, and also that the sulfate curves tend to have a less steep negative slope than the chloride curves. In addition, the lithium curve is consistently above the potassium curve for the chlorides, but the reverse is true for the sulfates.

\section{Discussion}

The data of this study appear to fit into the theoretical system suggested by Höber and Kiesow (1898)

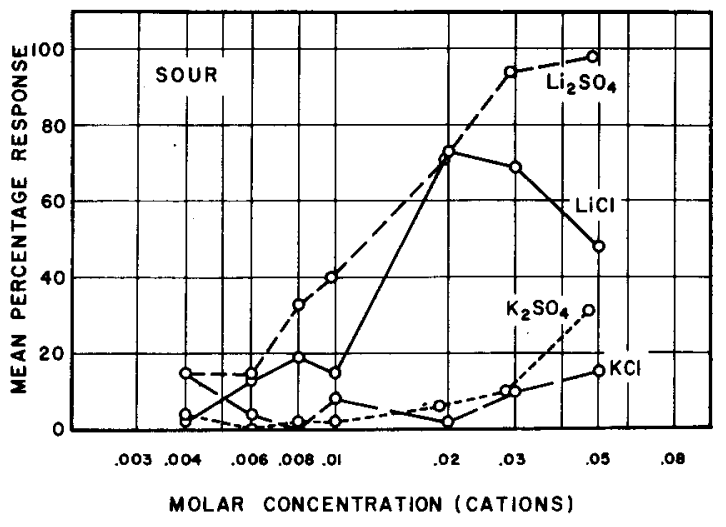

Fig. 3. The same type of plot as Fig. 2, except that these are sour responses.

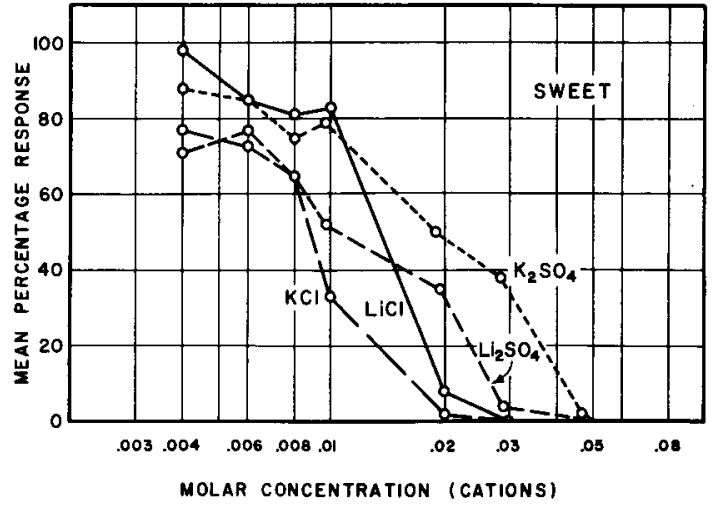

Fig. 4. Sweet responses, combined with those of no taste, plotted against cation concentration.

and by Kionka and Strätz (1922) in which the qualities of salts are effects of their separate lons, rather than into the scheme proposed by Renqvist (1919) who considered the qualities to result from the action of the salt as a whole. Specifically, the results are in agreement with the conclusions of Höber and Kiesow and of Kionka and Strätz that the chlorides and sulfates are responsible for the salty quality. The fact that lithium sulfate did not show an appreciable increase in this quality at higher concentration values used, is grounds for further testing of this salt at still higher concentrations to see if it does constitute an exception. The highest sulfate concentration in this experiment was approxdmately only half the chloride concentration.

The results indicated in Fig. 2 strongly reinforce the idea that it is the potassium ion which is responsible for the bitter quality of potassium salts. The sharp drop of the bitter responses to $\mathrm{KCl}$ above $0.02 \mathrm{M}$ is related to the sharp increase in salty responses above this concentration. The mechanism for this replacement of one gustatory quality by another which has a higher absolute threshold has been attributed earlier to an inhibitory phenomenon (Dzendolet \& Meiselman, 1967). The slower rise and the plateau exhibited by responses to potassium sulfate can, in the same way, be related to the fact that this sulfate was not able to stimulate the salty receptors too well, and this low level of stimulation was, therefore, insufficient to inhibit the bitter responses to the same degree as the chlorides did.

The data shown in Fig. 3 concerning the sour responses indicates that it is the lithium ion which is responsible for this quality in these salts. What has been said about the shapes of the curves for the potassium sulfate and chloride with regard to the bitter response can also be applied to the lithium salts, with merely a change in the name of the quality. The sour responses to lithium sulfate, however, are of a much greater percentage than the bitter responses to potassium sulfate. 
There is, however, a problem concerned with the assignment of sour responses to the lithium ion, because it is generally considered that the hydrogen ion is the structure responsible for evoking the sour quality. A possible basis for the explanation of this behavior of the lithium ion would be to invoke an interaction between the lithium ions and the water molecules of the solution, with a partial freeing of hydrogen ions as a result of this interaction. Harned and Owen $(1950$, p. 385) have described such an ion-solvent interaction as a 'localized hydrolysis." This localized hydrolysis should not be confused with the one suggested by Renqvist (1919) which was discussed earlier. These hydrogen ions would then be the source and cause of the sour quality, rather than the lithium ions themselves. It should be noted that the hypothesis given above is merely an initial statement of a suggested mechanism, and that a detalled description would require taking into account the role of other factors, e.g., the saliva. However, if it is assumed that the above hypothesis is correct, then it would be impossible to attribute a specific quality to the lithium ion itself because of this ion-solvent interaction.

As for the sweet quality, the data in Fig. 4 do not suggest that any one of the salts present is peculiarly responsible for the sweet quality. That is, all the salts seem to about equally effective in evoking the sweet quality except for the slight differences between them which were described earlier. If it is assumed that a specific structure should be responsible for evoking the sweet quality, it must then be hypothesized that such a structure should be present with respect to these salts. The following argument can be made as to whether the effect is caused primarily by the cation or the anion.
Because the slopes for the two chloride curves are similar to one another, as are those for the sulfates, but because the chloride and sulfate slopes are different, it is hypothesized that the cations are the primary cause for evoking the sweet quality, although the anions can modify their effectiveness. The most probable mechanism which, at low concentrations, would provide such diverse cations with a common structure, is in terms of an ion-solvent interaction of the kind that was postulated earlier for the lithium ion with respect to the sour quality. Because anions do not form such an interaction (Harned \& Owen, 1950 , p. 409), this would appear to lessen the probability that the anions might be primarily responsible, otherwise such vastly different anions as sulfate and chloride would have to be considered as being essentially equivalent structurally so as to produce their similar sweet-evoking effect. The exact nature of the structure resulting from such an ion-solvent interaction and its mechanism in evoking the sweet quality is yet to be determined.

\section{References}

Dzendolet, E., \& Meiselman, H. O. Gustatory quality changes as a function of solution concentration. Percept. \& Psychophys., $1967,2,29-33$.

Hamed, H. S., \& Owen, B. B. The physical chemistry of electrolytic solutions. (2nd ed.) New York: Reinhold, 1950.

Hobber, R., \& Kiesow, F. Ueber den Geschmack von Salzen und Laugen. Z. physikal. Chemie, 1898, 27, 601-616.

Kionka, H., \& Strätz, F. Setzt der Geschmack eines Salzes sich suzammen aus dem Geschmack der einzelnen Ionen oder schmeckt man jedes Salz als Gesamtmolekül? Arch. exp. Pathol. \& Pharmakol., 1922, 95, 241-257.

Renqvist, Y. Ueber den Geschmack. Skand. Arch. Physiol, 1919, $38,97-201$.

(Accepted for publication August 15, 1967.) 